ISSN: 2007-7033 | Núm. 54 | e1020 | Sección convocatoria: artículos teóricos temáticos (revisión sistemática) |

\title{
La formación profesional en turismo entre conexiones y redes de aprendizaje
}

\section{Professional education in tourism between connections and learning networks}

\author{
NANCY ORTEGA MARTIÑÓN* \\ JuANA LOURDES MEDINA CUEVAS ${ }^{* *}$ \\ MARIBEL OSORIO GARCÍA ${ }^{* * *}$
}

Los planteamientos vertidos en este artículo tienen como propósito argumentar las nuevas dinámicas de aprendizaje y escenarios para la formación en turismo, así como sus implicaciones en el ámbito profesional, que son favorecidos por la cultura digital. El estudio parte de la etnografía virtual e identifica redes de turismo en Latino-iberoamérica que propician mediaciones en una práctica educativa abierta y expansiva en la cultura digital. Los resultados se presentan con base en categorías de análisis y se concluye sobre las posibles transformaciones que han de gestarse en el ámbito educativo como un proceso de interactividad e interacción combinado entre los actores involucrados desde la academia y en la actividad turística.

The approaches expressed in this article are intended to argue the new learning dynamics and scenarios for training in tourism, as well as their implications in the professional field favored by digital culture, discerning about the trends and tensions of this era. Thus, a study is carried out from virtual ethnography identifying tourism networks in Latino-iberamérica that foster mediations in an open and expansive educational practice in digital culture. The results are presented from categories of analysis and it is concluded about the possible transformations that must be developed in the educational field as a process of combined interaction between the actors involved from the academy and in the tourist activity.

\section{Palabras clave:}

formación profesional, turismo, cultura digital, redes de aprendizaje, educación expandida

\section{Keywords:}

professional education, tourism, digital culture, learning networks, expanded education

Recibido: 29 de julio de 2019 | Aceptado para su publicación: 19 de febrero de 2020

Fecha de publicación: 28 de febrero de 2020

Recuperado de: https://sinectica.iteso.mx/index.php/SINECTICA/article/view/1020

doi: $10.31391 /$ S2007-7033(2020)0054-007

\footnotetext{
* Licenciada en Turismo por la Universidad Autónoma del Estado de México (UAEM). Profesora de la Facultad de Turismo y Gastronomía de la misma universidad. Línea de investigación: innovación educativa. Correo electrónico: ortega.nom@gmail.com

** Doctora en Educación por la Universidad La Salle, México. Profesora del Centro de Investigación y Estudios Turísticos de la Facultad de Turismo y Gastronomía de la UAEM. Líneas de investigación: innovación educativa y currículo en turismo. Correo electrónico: lumec57@gmail.com

${ }^{* * *}$ Doctora en Ciencias Sociales y Políticas por la Universidad Iberoamericana. Profesora del Centro de Investigación y Estudios Turísticos de la Facultad de Turismo y Gastronomía de la UAEM. Líneas de investigación: estudios sociales de la tecnociencia y estudios socioespaciales del turismo. Correo electrónico: maribelosorio2@gmail.com
} 


\section{INTRODUCCIÓN}

$\mathrm{L}$

a crisis que experimenta el tradicional paradigma educativo sacude sus principios por la configuración de escenarios alternativos al clásico entorno escolarizado. La inmersión en la cultura digital favorece las transformaciones en la manera de aprender de los individuos, que no implica, limitadamente, realizar un reajuste en estrategias, sino un cambio en la concepción del dónde y cómo aprender. Ante ello, surgen planteamientos pedagógicos adaptados a las circunstancias en las que se presenta el aprendizaje, lo que da pauta al reconocimiento de conceptos como aprendizaje ubicuo (Burbules, 2012), entendido como el que se produce en cualquier lugar y momento potencializado por medios digitales; edupunk (Groom, 2008), que alude a la enseñanza y el aprendizaje inventivos; aprendizaje invisible (Cobo y Moravec, 2011), relacionado con el conocimiento tácito y que tiene que ver con habilidades blandas o no cognitivas; o educación expandida (Díaz y Freire, 2012; Uribe, 2017, 2018); estos cobran mayor sentido en la cultura digital y aportan los fundamentos de nuestra investigación.

La formación en turismo no escapa a esta realidad y las renovadas directrices en el sector comprometen a reestructurar contenidos y escenarios de aprendizaje para preparar personas capaces de enfrentarse a los retos desencadenados por los rápidos cambios económicos, ambientales, sociales, culturales y tecnológicos a nivel mundial. Las tendencias internacionales a corto plazo (2030) definen la perspectiva del desarrollo humano como punto notable en el que se ha de consolidar el turismo. Una educación pertinente marcará la diferencia para el óptimo manejo de un destino o negocio turístico, por lo que se necesitará desarrollar capacidades para resolver problemas complejos, pensamiento crítico y creatividad (Osorio, Ramírez y Viesca, 2016), además de tener una fuerte conexión con la práctica de la industria digitalizada, personalizar sus rutas de estudio (Sterren, 2015) y fomentar nuevas formas de comunicación (Fernández, 2015).

Ante este escenario, la conectividad induce la creación de redes de aprendizaje (RA) como formas de interacción que llevan a una transformación educativa, pues gracias a ellas los implicados tienen la posibilidad de gestionar conocimiento en red en un ámbito abierto, flexible y articulador. Entonces, cabe preguntarse acerca de las oportunidades que conectan al profesional en turismo con un medio tan cambiante que reclama creatividad de sus estudiantes (Vázquez, 2015), que encierra el desarrollo de sus competencias culturales (Kereluik, Mishra, Fahnoe \& Terry, 2013; Sterren, 2015), y busca la personalización de su aprendizaje para fusionarse con su entorno (Sterren, 2015).

Por ello, es relevante discutir sobre las transformaciones y los nuevos escenarios a los que el turismo se enfrenta como profesión en la interconectividad regida por las RA en la cultura digital. El texto se compone de cuatro apartados: en el primero, puntualizamos las tensiones en la formación del profesional; en el segundo, delineamos la metodología aplicada para el estudio; en el tercero, exponemos los resultados de la revisión de experiencias sobre RA en turismo; y en el último, incluimos las reflexiones que, a manera de conclusiones, dilucidan los posibles escenarios de cambio de la formación en turismo. 


\section{TENDENCIAS Y TENSIONES EN LA FORMACIÓN PROFESIONAL EN TURISMO FRENTE A DINÁMICAS EDUCATIVAS EXPANDIDAS}

La tecnología ha cambiado la manera de hacer turismo; la fuerza que tiene sobre la actividad redefine modelos de negocios y procesos en su organización. En los últimos veinticinco años, la alianza entre tecnología y turismo se ha visto fortalecida por el exponencial desarrollo y acceso a internet en ordenadores y dispositivos móviles que han evolucionado desde la consulta de páginas web para la organización de un viaje hasta la creación de aplicaciones que fomentan la economía colaborativa reflejada en el alojamiento y el transporte.

No ajena a esta realidad, la Organización Mundial del Turismo (OMT), con el mandato de Zurab Pololikashvili, planteó cinco pilares que marcarían el trabajo de su gestión: "La innovación y la transformación digital; inversiones y emprendimiento; educación y empleo; viajes seguros y continuados; y sostenibilidad social, cultural y ambiental" (OMT, 2018a). Apostando a la innovación y la transformación digital, se celebró, en 2018, la primera edición de la Competición OMT de Startups de Turismo, en la que se expusieron proyectos innovadores con potencial de ser disruptores en el sector y pudieran cambiar la forma en que se viaja (OMT, 2018b).

El apoyo a este tipo de proyectos abona a la configuración del ecosistema traveltech o tecnología turística, que, además de abarcar el análisis, diseño, implementación y aplicación de soluciones tecnológicas al turismo, también tiene como objetivo satisfacer las necesidades de los viajeros del siglo XXI (Terrasa, 2018), el turista digital, caracterizado por estar hiperconectado, informado, acostumbrado al uso de apps y que busca interactuar para crear experiencias personalizadas.

Ante estas dinámicas que encara el turismo para la formación de profesionales, los desafíos se amplían para demandar personas que evolucionan con el cambio y desarrollan capacidades y habilidades para involucrarse con su medio. En tanto, estudiosos del tema, a lo largo de los años, han analizado esos retos, y la manera de aproximarse a cada situación se ha hecho más compleja según las circunstancias de los contextos. Damm (1998), Gómez (2005), García y Pérez (2008), Medina y González (2010), Ceballos, Arias, Ruiz, Sanz y Vázquez (2010), Fernandes (2011), y Díaz y Quiroz (2013) presentan un común denominador en sus escritos sobre el entendimiento de problemas que experimenta la educación en turismo y reflexionan sobre el papel del capital humano para el logro de objetivos de competitividad y diferenciación en las organizaciones turísticas.

A partir del entendimiento de las problemáticas expuestas, estos autores proponen profundizar en el desarrollo de competencias (técnicas, genéricas y específicas); el rediseño de planes de estudio; el incremento en el nivel educativo; ahondar en la proactividad y emprendedurismo; y que los estudios coincidan con las demandas laborales.

Por su parte, Carrera y Vallejo (2015), Montilla, Mora y Guzmán (2018) y Balakhadze (2018) señalan que los retos giran en torno al análisis de modelos de aprendizaje y a los procesos de enseñanza inherentes a la formación académica. Ejemplos de ello son los textos de Amador, Arroyo y Moo (2018), que evalúan un programa de estudios por competencia, y los de Contreras, Juárez y Ramírez (2018) y Manassero, Ramis, Ramírez y Guerra (2016), quienes valoran la 
percepción de estudiantes sobre las competencias en su formación académica profesional y su relación con el mercado laboral, así como sus expectativas, prioridades laborales y potencial directivo.

Algunas conclusiones generales derivadas del análisis, evaluación y diagnóstico realizados por estos últimos son:

-El turismo debe dar respuestas a temas globales.

-En el sector, el trabajo con las tecnologías de la información y la comunicación (TIC) es imprescindible y, por tanto, es necesario incorporarlas o fortalecerlas en planes de estudio.

- Hablar más de nuevas competencias que de nuevos puestos de trabajo, es decir, los perfiles profesionales tradicionales no son estáticos, sino dinámicos.

Tomando como referencia los documentos anteriores, identificamos que el avance en este tipo de publicaciones respecto a los requerimientos a superar para la formación en turismo se ha dado en dos sentidos: por un lado, aquellos escritos que exponen los escenarios a los que se enfrenta la formación y describen y reflexionan sobre las situaciones en las demandas educativas y laborales; y por otro, aquellos que, a partir de diagnósticos y evaluación, realizan propuestas para coadyuvar al mejoramiento de la formación académica. No queda exento, en ambos casos, hablar de competencias; sin embargo, una diferencia marcada es que en el segundo ya se aluden los impactos que las TIC tienen para la formación, algo ausente en el primer grupo de textos.

Una tercera línea (Dachary y Arnaiz, 2016; Gómez, 2010) busca redefinir la concepción tradicional del turismo desde los discursos educativos y del quehacer científico como referente de modelos educativos críticos, innovadores, integradores, con compromiso social y ético.

Este planteamiento general de publicaciones sobre la formación en turismo da pauta a reconocer un panorama distinto en el que hay que ser enfáticos: es insuficiente adentrarnos en los problemas y retos para la formación en turismo a partir de lo que el sector exige o de las competencias que los profesionales deben adquirir, puesto que estas se modificarán en el tiempo con dinámicas diversas; por ello, es preciso concebir un escenario alternativo a la formación académica en el que la apertura a innovadoras formas de comunicación y de consumo, la diversificación de los productos turísticos y las variaciones en la estructura de la sociedad contribuyan a involucrarse en nuevos modos de aprender.

Marquemos distancia del cliché que dentro de la tercera revolución industrial la educación ha sufrido cambios en sus escenarios de aprendizaje a consecuencia de la implementación de las TIC; consideremos, preferiblemente, que existe un trasfondo en la manera en la que se adquiere y gestiona nuevo conocimiento dentro de un mundo conectado; ya no basta ni es exclusivo aprender de modo institucional; se "necesitan nuevas organizaciones e ideas creativas y viables que reemplacen los métodos obsoletos de enseñanza y las relaciones autoritarias entre los actores participantes" (Gómez, 2005, p. 40).

La obligada revitalización de la educación por efecto de la tecnología digital y del internet rompe con los límites formales e institucionales de la escuela (Uribe, 
2018); se abren escenarios de formación tangibles e intangibles y se constituye un movimiento alternativo que repiensa los planteamientos críticos de la escuela y concuerda con las propuestas asociadas a la cultura digital y a la Web 2.0 (Uribe, 2017): la educación expandida, la cual sostiene que "la educación ya sucede, sobre todo, fuera de las instituciones educativas y de los procesos educativos formales" (Díaz y Freire, 2012, p. 71).

En esta perspectiva, el aprendizaje es por el bien común y se caracteriza por ser colaborativo, distribuido, abierto y experimental. Los límites entre el aprendizaje formal y no formal se vislumbran más tenues e, incluso, estos conceptos comienzan a ser anticuados para referirse a los fenómenos educativos surgidos dentro de la cultura digital.

El aprendizaje peer to peer (p2p), entendido como el aprendizaje entre pares, la autoformación, la creación colectiva y la conexión con fuentes de información especializada empoderan a los usuarios y los adentra cada vez más en la educación expandida al volverse prosumidores educativos inmersos en una cultura participativa en la que se hacen presentes RA, las cuales desarrollan conocimiento de manera colaborativa con efectos de mayor alcance en el aprendizaje, que contribuye a la mejora e incremento de las posibilidades de acceso de los implicados a un cúmulo de experiencias y materiales.

Solórzano y García (2016) exponen que las RA, además de ayudar a los participantes a desarrollar competencias colaborando y compartiendo información, enriquecen la experiencia de aprendizaje en contextos de educación no formal y formal, y son puntuales al especificar las herramientas de apoyo, como blogs, wiki, podcast, RRS, slide share, YouTube, Twitter, entre otros.

Desde esta perspectiva cambia la manera de pensar en la formación en turismo, puesto que no basta con exponer los problemas a los que se enfrenta a partir de las competencias ausentes en el profesional o de la poca pertinencia que tienen los planes de estudio en distintas universidades. Tampoco se niega que investigaciones que han abordado estos puntos aporten testimonios benéficos para la comunidad académica, pero los planteamientos ya comienzan a quedar rebasados por la necesidad de considerar las nuevas dinámicas dentro de la cultura digital que nos hacen encontrar distintas formas de acceder al conocimiento, de cómo construirlo y cómo aplicarlo en un campo multidimensional como el del turismo.

La formación profesional en turismo, desde este ángulo, ya no se vuelve exclusiva de la educación institucionalizada; comienza a constituirse un nuevo ente que va más allá de la escolarización. Esta formación puede acontecer en el ámbito académico, en espacios híbridos, con dinámicas de autoformación, creación colectiva y comunicación con diversas personas u organizaciones. No se excluye por completo a la educación formal, pero ya no es eje central del aprendizaje que va a ocurrir en condiciones sociales y culturales, como un fenómeno característico de la época.

Al respecto, Medina, Collado, Ortega y Arango (2012) ya planteaban la propuesta de RA para el turismo como una estrategia pedagógica que promoviera el aprendizaje en red; destacan que "quienes estén involucrados en el turismo, puedan comunicar, informar, realizar eventos conjuntos, desarrollar proyectos de diversa naturaleza y desarrollar propuestas educativas" (p. 11). Su proposición de RA busca fomentar el 
trabajo colaborativo y un aprendizaje dinámico no pensado en un aprendizaje a distancia, sino en uno complementario al trabajo presencial.

Frente a las versátiles directrices de la educación y del turismo, cabe entonces cuestionarse sobre experiencias de aprendizaje generadas por la educación expandida que han permitido la conformación de RA en la cultura digital en la que los enlaces surgen por relaciones simbólicas.

\section{EDUCACIÓN EXPANDIDA, CULTURA DIGITAL Y REDES DE APRENDIZAJE}

La educación expandida logró romper la idea dominante de que la escuela es el espacio exclusivo del saber; el aprendizaje dejó de construirse privilegiadamente en los límites formales institucionales y comenzó la transición de una sociedad con un sistema educativo tradicional hacia una que gestiona su propio aprendizaje y conocimiento dentro de una cultura digital que va constituyendo una diferente ecología del aprendizaje.

Coll (2013) y Barron (2006) ya trazaban una ecología que planteaba retos inéditos en la educación escolar. Para Coll, esta ecología del aprendizaje contrastaba con la que está vigente en los sistemas educativos actuales con protagonismo absoluto de la educación formal y escolar. Sostiene que cambios sociales, económicos y culturales trastocan los entornos y recursos para aprender y estos afectan a todos los parámetros del aprendizaje: el dónde, cuándo, qué, para qué, con quién, y, sobre todo, el cómo se aprende.

Coll (2013) es puntual en especificar que algunos rasgos de la ecología tienen especial relevancia en el currículo escolar, entre ellos la importancia del aprendizaje a lo "largo" y "ancho" de la vida; la tendencia a la informalización del aprendizaje; la multiplicidad de escenarios y agentes educativos y, por último, la aspiración a una personalización creciente del aprendizaje que remite a la singularidad de las trayectorias personales de formación. Asimismo, reconoce que existe un desdibujamiento del sentido de la educación escolar y cuestiona si aún cumple con las expectativas y finalidades atribuidas tradicionalmente.

A pesar de que Coll está enmarcando todo su planteamiento en el currículo escolar, sus ideas nos acercan a reflexionar en "otras" ecologías de aprendizaje; no podríamos asignarles con contundencia el adjetivo de "nuevas", pues las transformaciones tecnológicas y sociales nos hacen caer en la obsolescencia con rapidez; más bien, se trata de ecologías de aprendizaje cambiantes en las cuales la destemporalización, la deslocalización, la descentralización, y la pertinencia e interconectividad del aprendizaje la caracterizan.

Las ecologías de aprendizaje en la perspectiva de la educación expandida inciden en la manera en que se ha modificado la forma de aprender en la era digital, la cual tiene un acentuado enfoque conectivista en virtud del tipo de enlaces que se generan para lograr propósitos educativos. De acuerdo con los principios del conectivismo, el aprendizaje depende de la conexión entre nodos especializados y de la diversidad de opiniones entre las fuentes de información (Siemens, 2004); por ello, la identificación de conexiones entre diferentes áreas, ideas y conceptos se vuelve una habilidad clave para la actualización del conocimiento, que es la intención de todas las actividades conectivistas de aprendizaje. 
El vínculo establecido entre nodos remite a la conformación de RA, pero no de aquellas RA que planteaban Harasim, Hiltz, Turoff y Teles en el 2000, con una faceta exclusivamente escolarizada, sino a las que son vistas a través de la educación expandida. La intervención de las RA desde la lógica expandida da pauta a que se estructure un enfoque compartido a partir de las interconexiones, intercambio de saberes, experiencias y posibilidades de desarrollo de cada uno de los integrantes que las conforman, quienes no son, de modo necesario, alumnos y profesores.

Estas redes conectan "prácticas sociales distantes geográficamente y comunican de manera más rápida y flexible, nodos dispersos" (Uribe, 2015, p. 185); de igual modo, representan claras manifestaciones del individuo como productor de conocimientos con la capacidad de establecer alianzas entre diversos sectores; esto, aunado a su necesidad de transferir e intercambiar lo que crea y aprende. Considerándolas en la educación expandida, las RA no son limitativas al aprendizaje en línea; la propiedad que tienen de ser estructuras dinámicas permite también el vínculo en forma presencial; no obstante, su impacto es exponencial en la virtualidad y su fortalecimiento e incremento ha sido respaldado por las TIC y el internet.

En el ciberespacio, concibiéndolo como un contexto social y colectivo de comunicación en línea, las RA han pasado de ser discursos lineales entre emisores y receptores a comunidades con conciencia desde una cognición social. Su crecimiento corresponde en paralelo al del ciberespacio y, según Lévy (2007), tres principios orientaron su crecimiento (desde el más elemental al más elaborado): la interconexión, la creación de comunidades virtuales y la inteligencia colectiva. En su conjunto, estos principios son fundamentales en la cultura digital, además de los "valores, normas, prácticas y expectativas compartidas con respecto a la forma en que la gente actúa e interactúa dentro de la sociedad red contemporánea" (Deuze, 2006, p. 63).

Las RA inmersas en la cultura digital son modificadoras en las prácticas de producción de conocimiento y contribuyen a la ruptura del paradigma clásico de aprendizaje, asociado a los principios de la educación expandida que, como lo sostiene Fonseca (2011), es más un ethos que una metodología y emerge como una forma de activar prácticas innovadoras, enfoques por problemas vitales y transversales a la sociedad al inaugurar una expansión en las formas de hacer y pensar los procesos de formación. Entonces, de acuerdo con Lévy (2007), si los individuos están aprendiendo de sus propias experiencias sociales y profesionales, y las instituciones pierden, gradualmente, el monopolio de creación y la transmisión del conocimiento, es preciso reorientar los recorridos individuales en el saber y aportar al reconocimiento del conjunto de los saberes adquiridos por las personas, incluidos los no académicos.

En este punto, cabe reflexionar que, al expandir el entorno de aprendizaje por medio de las RA, una persona se involucra en una dinámica diferente en la que se apoya de herramientas propias de una cultura digital y configura espacios que le permiten darle sentido a la realidad desde la que está interactuando. Esta interacción es un referente de partida para conocer si la cultura digital ha transformado la formación profesional en turismo ante los nuevos modos de aprender que surgen en una conectividad integrada mediante RA, las cuales fluyen en el ámbito de la profesión, dadas sus dinámicas cambiantes en las que convergen inéditos campos de interrelación. 
Si bien la interacción es un punto de partida, no es el único factor que determina transformaciones dentro de la cultura digital. Las características de las RA desde la educación expandida y los principios de crecimiento del ciberespacio propuestos por Lévy se abordan en el estudio a partir de categorías y subcategorías que facultan la comprensión de hechos ocurridos en espacios digitales, que consideran los principios y las propiedades de las RA que son susceptibles de análisis (ver tabla 1).

Tabla 1. Categorías y subcategorías

\begin{tabular}{|c|c|}
\hline Categoría & Subcategoría \\
\hline \multirow{2}{*}{ Interconexión } & Interacción en las RA \\
\cline { 2 - 2 } & Establecimiento de alianzas entre diversos sectores \\
\cline { 2 - 2 } Comunidades virtuales & Configuración de vínculos sociales \\
\hline \multirow{3}{*}{ Inteligencia colectiva } & Creación colectiva \\
\cline { 2 - 2 } & Desarrollo de conocimiento colaborativo \\
\cline { 2 - 2 } & Intercambio de saberes \\
\hline
\end{tabular}

Fuente: Elaboración propia con base en Lévy (2007).

La descripción de las categorías sugiere un acercamiento epistémico detallado; no obstante, retomamos los apuntes centrales de cada una de ellas:

-La interconexión, que establece la relación entre los participantes que sucede con frecuencia y logra establecer vínculos. En ella se identifican tres subcategorías: la interacción que fortalece la red, referida a la comunicación interpersonal mediada por tecnología ocurrida de modo sincrónico o asincrónico; establecimiento de alianzas entre diversos sectores, como el público, privado, social o académico que habilitan la asociación entre redes; y la configuración de vínculos sociales que pueden darse de manera endógena y exógena en la red.

- Las comunidades virtuales, consideradas también como redes, son agregados sociales constituidos sobre afinidades de intereses y de conocimiento; comparten proyectos en un proceso de cooperación e intercambio que deriva en la creación colectiva a partir de la conectividad de todos los miembros.

-La inteligencia colectiva, que comprende el reconocimiento y enriquecimiento mutuo de las personas a partir de la colaboración abierta en línea. El desarrollo de conocimiento colaborativo es la primera subcategoría que refleja la participación activa de los integrantes de la red; a mayor pasividad, menor desarrollo de conocimiento colaborativo. La segunda subcategoría, intercambio de saberes, determina si existe reciprocidad entre los integrantes de la red o tienden al unilateralismo. Finalmente, producción de conocimiento, refleja aquellos productos generados al interior de la red derivados del trabajo colaborativo.

A partir de la categorización que orienta el estudio, reconocemos experiencias que han transformado y favorecido distintos modos de aprender en la cultura digital en proximidad con la formación profesional en turismo; en el siguiente apartado, presentamos los resultados de investigación que dan cuenta de cómo las RA son generadoras de interacción, conexión y colaboración en entornos virtuales. 


\section{EXPERIENCIAS DE REDES DE APRENDIZAJE EN TURISMO. UN ACERCAMIENTO DESDE LA ETNOGRAFÍA VIRTUAL}

El enfoque metodológico que guía la investigación es la etnografía virtual (Hine, 2004), que sitúa a los objetos de estudio en dimensiones que ya no son físicas, sino virtuales impulsadas por vínculos sociales, y transitan al plano de las relaciones en las que los procesos de interacción son la base para la interpretación de las comunidades en internet (Álvarez, 2009). Con base en esta metodología, Hine propone dos formas de estudiar el internet: como cultura y como artefacto cultural. En nuestro estudio, retomamos la primera propuesta en la que se ve el internet como el lugar donde se producen las interacciones que permiten la conformación de una cultura y donde se hace hincapié en el análisis del contexto y en los discursos que se producen entre los miembros.

El proceso etnográfico en un entorno digital, de acuerdo con Sánchez y Ortiz (2017), implica que, una vez delimitado el problema de investigación, se planea el ingreso a la comunidad; posteriormente, se realiza el trabajo de campo y se analizan los resultados. Para estos autores, el proceso etnográfico es abierto, de tal modo que permite retornar y ajustar los límites del problema y detallar información necesaria para la descripción de la comunidad.

A diferencia de la etnografía tradicional, establecer un espacio de estudio en red representa cierta complejidad, pues implica ir más allá de seleccionar geográficamente un entorno y considerar las relaciones que en red se generan para dar un seguimiento continuo. Álvarez (2009) especifica que la definición de la comunidad se precisa en el curso de la investigación y no a priori, lo que ocurrió en el transcurso de la investigación; no obstante, en un principio, determinamos criterios de elección para delimitar el tipo de redes como comunidades a observar y particularizamos en entornos abiertos de lengua española en la región latino-iberoamericana que hicieran uso de herramientas de comunicación representativas de la Web 2.0 con presencia en la temática del turismo.

La observación no participante como técnica etnográfica nos permitió la identificación de espacios apropiados y favoreció la figura de lurker activo, con lo que obtuvimos información de la comunidad que utilizamos para el registro etnográfico.

Como punto de partida para la selección del entorno, elegimos comunidades abiertas de estudiantes en turismo adscritos a una educación formal; esta opción podría parecer contradictoria al discurso que se ha manejado en este documento, pero nos dio pauta para identificar vínculos con otro tipo de redes, lo que llevó a que los entornos estudiados fueran cambiando de acuerdo con las conexiones que se iban enlazando o desconectando a lo largo de la investigación.

Derivado de lo anterior, el tipo de redes identificadas fue variado; algunas de ellas producidas de investigaciones, proyectos universitarios o gubernamentales, o aquellas que surgieron de la necesidad de comunicación entre participantes para compartir experiencias personales sin alguna intencionalidad académica formal. Con base en los criterios de selección, optamos en un primer periodo de observación (2016-2017) por siete redes. En la tabla 2 mostramos, de manera sintetizada, su evolución en el primer periodo del estudio. 
Tabla 2. Síntesis del seguimiento a las redes seleccionadas en el primer periodo

\begin{tabular}{|c|c|c|}
\hline Red & País & Seguimiento \\
\hline Red de estudiantes de Turismo & Argentina & $\begin{array}{c}\text { Desapareció por falta de acceso libre a la plataforma } \\
\text { que la albergaba }\end{array}$ \\
\hline $\begin{array}{l}\text { Red de alumnos del máster oficial } \\
\text { en Dirección y Planificación Turís- } \\
\text { tica de la Universidad de Alicante }\end{array}$ & España & $\begin{array}{l}\text { Tuvo actualizaciones continuas en sus páginas, pero } \\
\text { pasado un tiempo se suspendieron sus colaboraciones }\end{array}$ \\
\hline $\begin{array}{l}\text { Red social para alumnos de } \\
\text { Turismo de la UNED }\end{array}$ & España & $\begin{array}{c}\text { Se alojaba en plataforma tipo CMS y luego migró a la } \\
\text { red social Facebook; continúa vigente por la necesidad } \\
\text { del intercambio de información por parte de los alum- } \\
\text { nos que estudian el grado de Turismo. En Facebook se } \\
\text { han creado grupos cerrados de las asignaturas de la } \\
\text { carrera en los que los alumnos se inscriben mientras } \\
\text { estén cursando la asignatura y, una vez acreditada, la } \\
\text { mayoría se dan de baja para ingresar a otros grupos } \\
\text { que les corresponden }\end{array}$ \\
\hline Red de estudiantes de Turismo & Perú & $\begin{array}{c}\text { Se quedó como propuesta de proyecto sin desarrollo } \\
\text { posterior }\end{array}$ \\
\hline $\begin{array}{l}\text { Red Internacional de Investiga- } \\
\text { dores en Turismo, Cooperación y } \\
\text { Desarrollo, Coodtur }\end{array}$ & España & $\begin{array}{l}\text { Continúa fortaleciendo su trabajo académico y de } \\
\text { investigación }\end{array}$ \\
\hline $\begin{array}{l}\text { Red Hispano Lusa de Investigado- } \\
\text { res en Turismo, Reintur }\end{array}$ & España Portugal & $\begin{array}{l}\text { Dejó de actualizar información en su blog; pese a } \\
\text { ello, los miembros mantienen contacto por medio de } \\
\text { Facebook en un perfil cerrado creado para tal fin }\end{array}$ \\
\hline $\begin{array}{l}\text { Red Universitaria de Posgrados en } \\
\text { Turismo Redintur }\end{array}$ & España & $\begin{array}{c}\text { Continúa fortaleciendo su trabajo académico y de } \\
\text { investigación }\end{array}$ \\
\hline
\end{tabular}

De las siete, elegimos dos redes para profundizar en su observación: Coodtur y Redintur, debido a que continuaban con su trabajo colaborativo a nivel nacional e internacional; en ellas son partícipes investigadores y estudiantes de posgrado en el área turística.

La aproximación en este primer periodo a estas redes nos permitió perfeccionar la búsqueda para un segundo periodo (2017-2019), durante el cual tomamos en cuenta dos nuevos criterios de selección: escenarios de aprendizaje en sus diversas posibilidades: educación formal e informal, acción sociocomunitaria, educación permanente o web social (Rivas-Flores, Martínez, ValverdeBerrocoso y Fernández-Rodríguez, 2018); y entornos que reflejaran procesos de creación y gestión de conocimiento práctico. En consecuencia, identificamos Entorno Turístico de México, Campus Mintur de Argentina, Aprende de Turismo de España, Red Turismo Chile y Hosteltur, también de España. Estas cinco autonombradas "plataformas" han concretado su trabajo y presencia gracias a la colaboración continua desde hace varios años.

Con la intención de tener un mejor acercamiento para la observación de las comunidades, fue necesario registrarse en cuatro de ellas a fin de determinar sus reglas y normas de comunicación y darles un seguimiento oportuno. El registro es un requisito indispensable si se desea publicar o tener acceso a contenidos exclusivos como boletines, cursos en línea, ofertas laborales o publicaciones especiales; además, 
permite conocer el perfil de los participantes que interactúan dentro de ella; así, pudimos saber que los miembros son estudiantes, recién egresados, investigadores, analistas, profesores, empresarios y personas que, sin tener estudios precedentes en turismo, están inmersos en el área.

El seguimiento a las demás redes se realizó directamente en su página oficial y en redes sociales; el acceso abierto a la información proporcionada en sus sitios web hizo posible que distinguiéramos perfiles de usuarios semejantes a las anteriores, excepto analistas y empresarios.

Además de la actualización constante en sus plataformas, las comunidades observadas publican con frecuencia contenido en redes sociales como Facebook y Twitter; de igual manera, se apoyan de otras redes, como LinkedIn, Pinterest, Instagram o YouTube para contenido específico; sin embargo, las más usuales son las dos primeras. Para dar seguimiento y detallar la observación etnográfica, optamos por Twitter, que da cuenta de la relación de las comunidades seleccionadas.

Como resultado de la búsqueda y seguimiento en los dos periodos de la investigación, elegimos siete redes para su observación y posterior análisis. Respetamos los derechos autorales y cuestiones éticas que tenían que ver con el contenido de las RA; solicitamos, vía correo electrónico, la colaboración de cada comunidad y red estudiada para el uso de datos disponibles de acceso abierto relacionados con la red. Las respuestas de los presidentes, gerentes, directores y editores responsables fueron positivas y aprobaron el uso de dichos datos.

\section{Análisis de los resultados}

El análisis de las RA como comunidades se sustenta en las categorías y subcategorías que orientaron la observación etnográfica virtual del estudio; de acuerdo con las particularidades de las subcategorías, realizamos el acercamiento a cada red para examinar su comportamiento. Como resultado de la observación virtual en el trabajo de campo, identificamos las actividades de las redes y establecimos sus relaciones internas que configuran ecologías de aprendizaje a partir de sus intencionalidades y formas de construirse; así, logran organizarse como redes portadoras de aprendizaje expansivo. A su vez, distinguimos las potencialidades de cada red y los vínculos entre ellas.

\section{Hallazgos}

Para detallar las observaciones a partir del trabajo de campo, presentamos de manera sintética los resultados por cada red observada. Para ello, hacemos una descripción general de las RA y su análisis con base en las particularidades de las subcategorías. 


\section{- Coodtur (http://www.coodtur.org/)}

Es una plataforma académica de difusión y transferencia para los estudios e investigaciones sobre turismo y desarrollo para acercar la academia y las acciones de cooperación al fomento turístico. Desde sus inicios, la red ha crecido considerablemente en relación con su interconexión. La adhesión de miembros permite una mayor interacción entre investigadores de diferentes nacionalidades de reconocida trayectoria en el turismo. Los grupos de investigación y organizaciones a los que pertenecen algunos permiten establecer alianzas entre diversos sectores, y sus vínculos sociales se han fortalecido en los seminarios y congresos realizados en España, México y Chile.

Trabajar en proyectos en conjunto ha fortalecido la producción y el desarrollo de conocimiento colaborativo con productos como artículos y libros. La red se ha consolidado en la interconexión y el fomento de inteligencia colectiva por la experiencia y el conocimiento de sus integrantes; no obstante, por el propio perfil de la red, la apertura hacia otro tipo de miembros que no sean investigadores o personas adscritas a una comunidad académica limita las posibilidades de ampliar espacios de aprendizaje para aquellos interesados en fortalecer su formación en turismo; no negamos que los productos que la red genera aportan conocimiento a otras personas, pero existe débil interacción y promoción de conocimiento colaborativo fuera de ella.
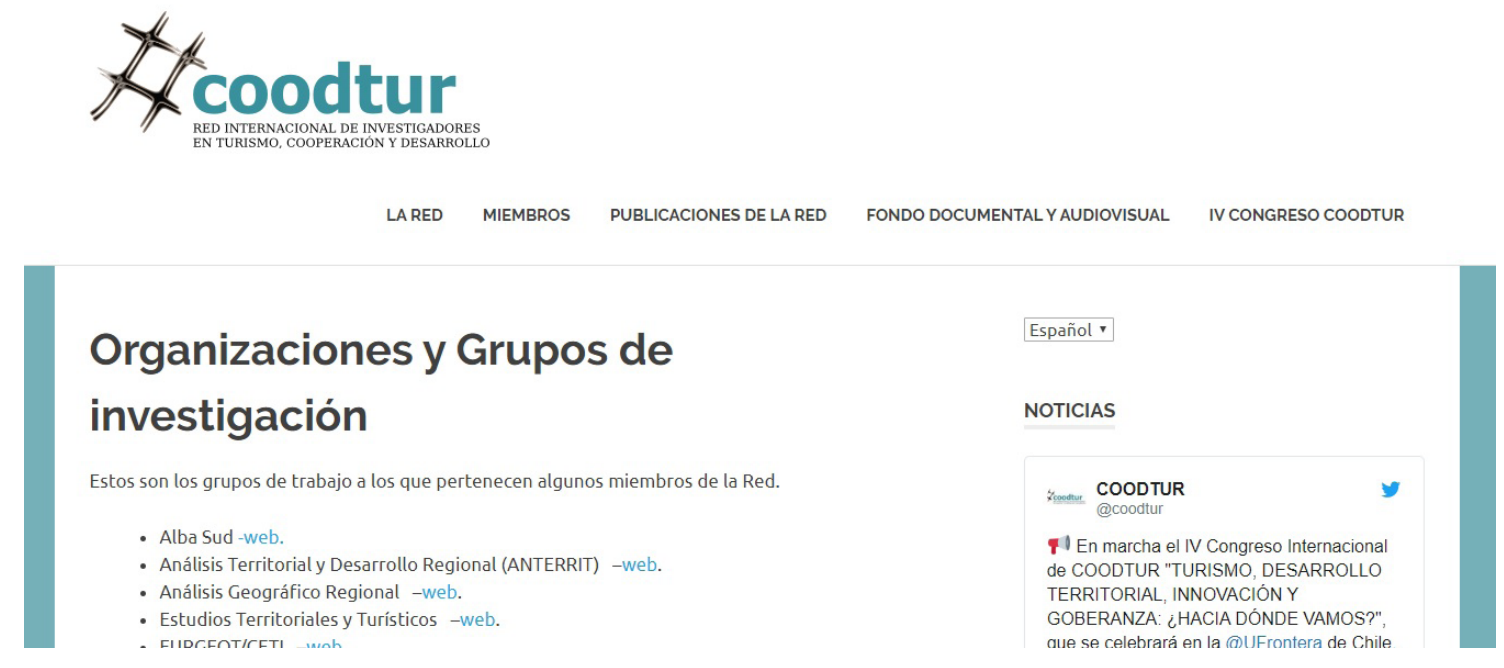

Figura 1. Miembros de Coodtur. Fuente: http://www.coodtur.org/membres/grups-dinvestigacio/ 


\section{- Redintur (https://red-intur.org/)}

Red conformada por 28 universidades españolas que buscan la cooperación en la docencia de posgrados; el fomento, intercambio, movilidad entre profesores y estudiantes; la realización conjunta de proyectos y la transferencia de conocimiento turístico. El beneficio de la interacción generada en su interior favorece a estudiantes de posgrado y a docentes de las universidades que son miembros, lo que fortalece la interacción.

A través de los Foros Redintur, que son un espacio de debate sobre la formación de posgrado e investigación en turismo en los que se presentan los mejores trabajos de fin de máster, se han configurado vínculos sociales exógenos y se ha establecido un intercambio y generación de conocimiento turístico mediante mesas redondas y aportaciones de expertos e investigadores del sector. El conocimiento derivado se publica en su boletín mensual y en revistas científicas con alto factor de impacto, lo que beneficia la producción de conocimiento.
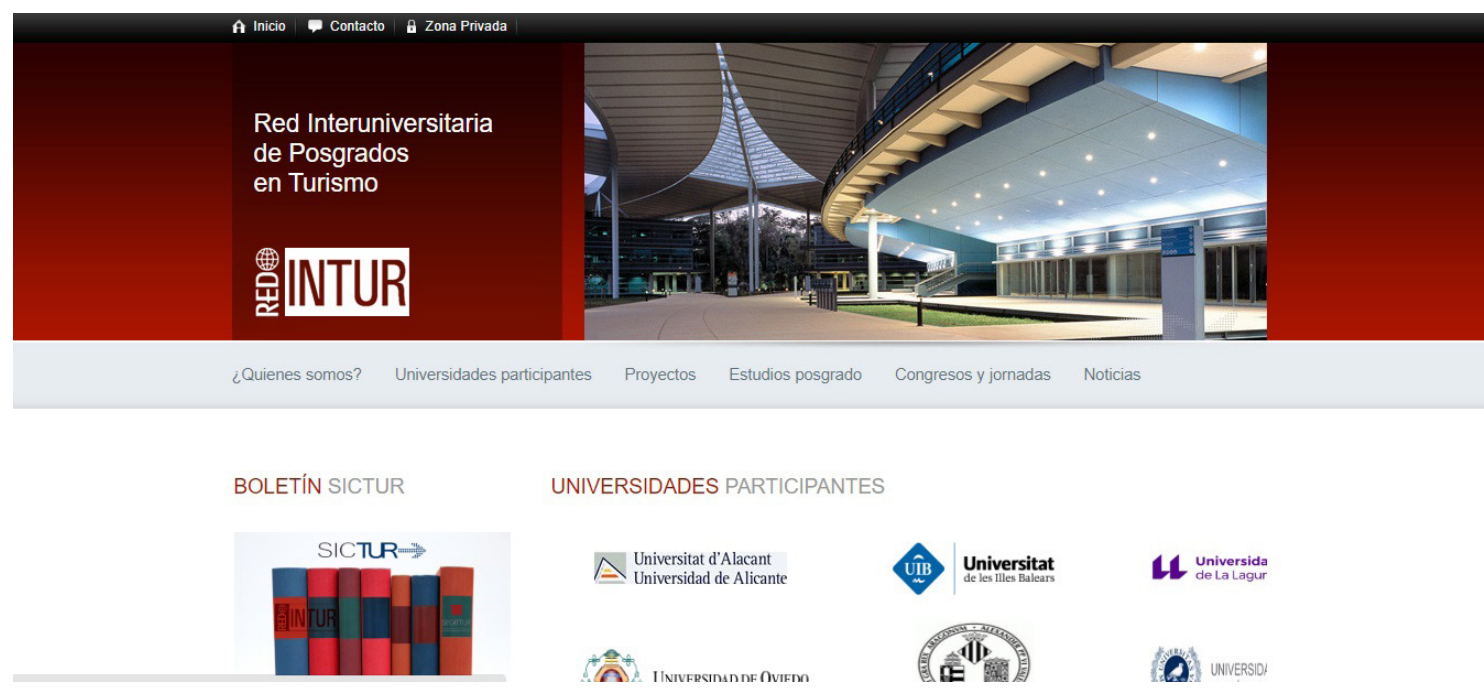

Figura 2. Universidades adscritas a Redintur. Fuente: https://red-intur.org/participantes.html 
- Aprende de Turismo (https://www.aprendedeturismo.org/)

Proyecto creado en 2014 para ofrecer un programa formativo en línea enfocado a profesionales del sector turístico de todo el mundo que buscan mejorar sus competencias laborales; no se encuentra vinculado a alguna institución educativa y ofrece cursos gratuitos y de pago. Los miembros ven una oportunidad de empezar, continuar o concluir con su formación en turismo, la interacción y vínculos sociales surgen de la necesidad de los propios usuarios para consulta y fortalecimiento de conocimientos sobre algún tema específico.

Por la naturaleza de la red, el desarrollo de conocimiento colaborativo es parcialmente participativo, pues los contenidos ya se encuentran predeterminados y el intercambio de saberes tiende a ser unilateral; no obstante, al interior de la red siempre existe una retroalimentación por parte del responsable del curso, que apoya en los foros de discusión o da respuesta a comentarios y mensajes directos. Además de los cursos, Aprende de Turismo cuenta con un blog que publica artículos o noticias de vanguardia que reflejan su destacada producción de conocimiento, los cuales son promovidos en su cuenta de Twitter.

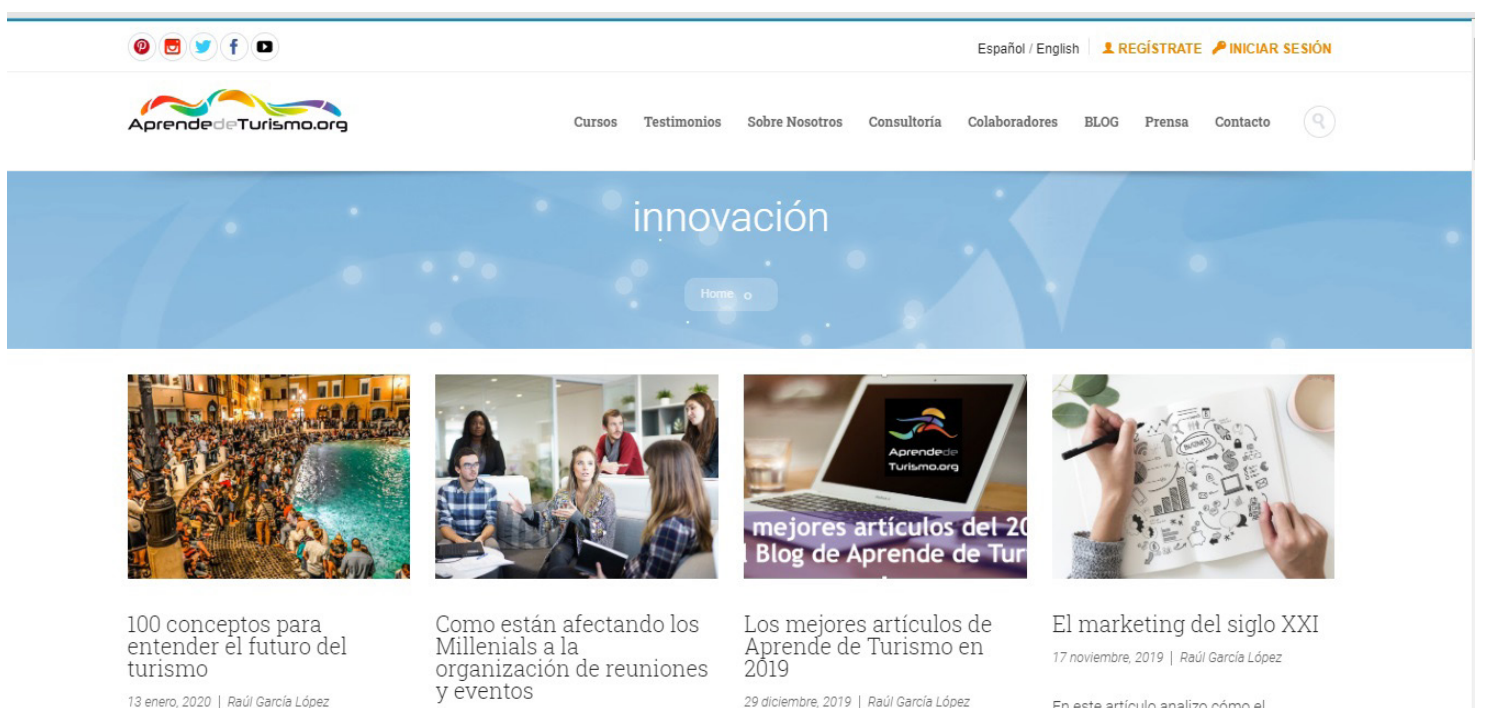

Figura 3. Blog de Aprende de Turismo. Fuente: https://www.aprendedeturismo.org/tag/innovacion 


\section{- Entorno Turístico (https://www.entornoturistico.com/)}

Medio en línea de comunicación turística creado en 2015. Dedica sus contenidos a estudiantes, académicos, empresarios, prestadores de servicio e instituciones públicas para informar del acontecer del medio turístico a nivel local e internacional y divulgar conocimiento para la comunidad, con lo cual logra una destacada asociación de alianzas y configura vínculos exógenos. Brinda información del turismo a través de artículos, noticias, investigaciones o columnas de opinión creados por colaboradores de distintas nacionalidades especializados en diversos temas, quienes comparten sus conocimientos y experiencias.

Lo anterior ha hecho de Entorno Turístico un espacio de creación colectiva de amplia conectividad y destacada producción de conocimiento, que es su pilar fundamental que la posiciona como un medio de comunicación influyente en México. Promueve la interacción y participación de los miembros para lograr conocimiento colaborativo e invita a colaborar con publicaciones a través de su página oficial. En redes sociales se refleja la fuerte interacción que obtiene al promover publicaciones divulgadas en su página.
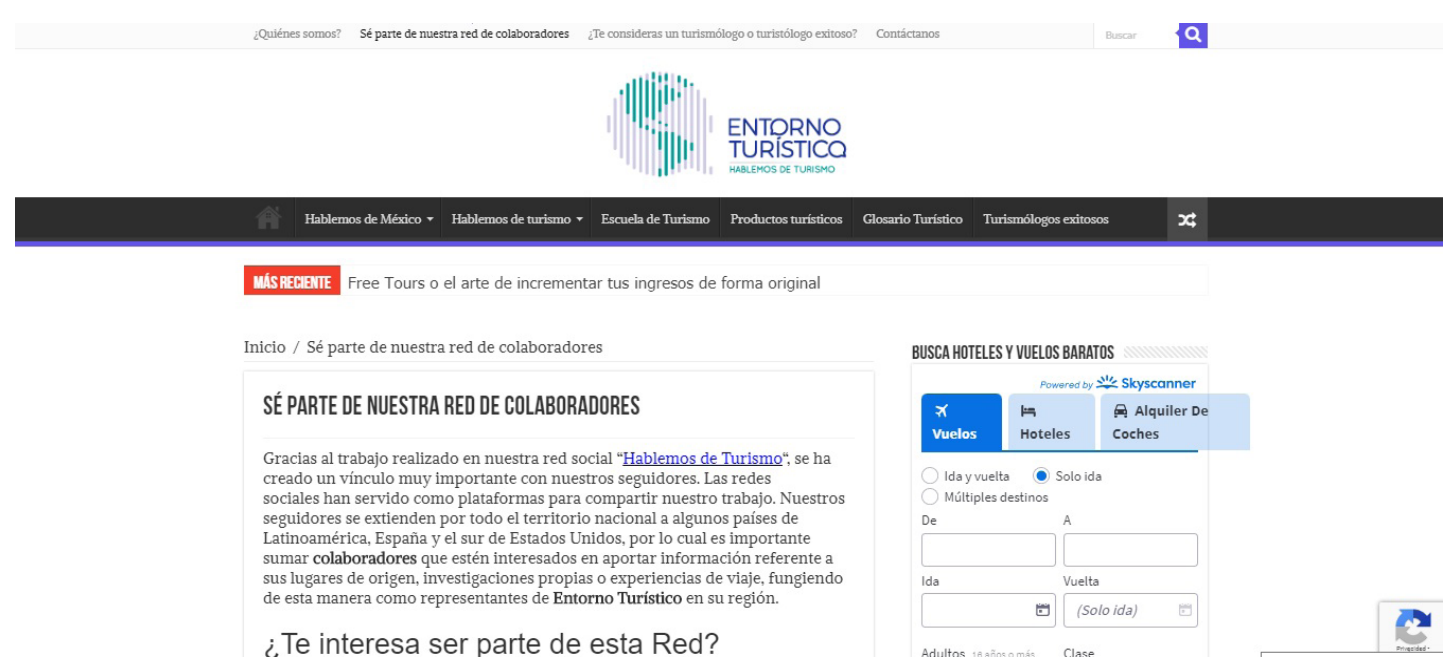

Figura 4. Requisitos de colaboración para Entorno Turístico. Fuente: https://www.entornoturistico.com/separte-nuestra-red-colaboradores/ 
- Campus Mintur (https://campus.yvera.gob.ar/)

Impulsado por la Secretaría de Gobierno de Turismo de Argentina (Ministerio de Turismo de la Nación), esta red ha ofrecido, desde 2011, capacitación virtual para dar respuesta a las necesidades de la comunidad turística, tanto del sector público como privado, a través de cursos virtuales que permitan el desarrollo de competencias para buscar la mejora del desempeño en el puesto laboral o profesional. Al acceder a la plataforma, se puede matricular a cursos virtuales autogestionados, de idiomas o con tutor.

La interacción dentro de la plataforma ocurre con los tutores o con otros miembros interesados en determinado tema, pero no es una condición fundamental de la comunidad, lo que podría debilitar su interacción. La configuración de los vínculos sociales es propensa a ser endógena; por ello, el intercambio de saberes tiende a la unilateralidad; en consecuencia, observamos una pasividad en el desarrollo de conocimiento colaborativo y limitada producción entre los miembros registrados; no obstante, en redes sociales hay mayor participación y vínculos sociales fuera del sitio.
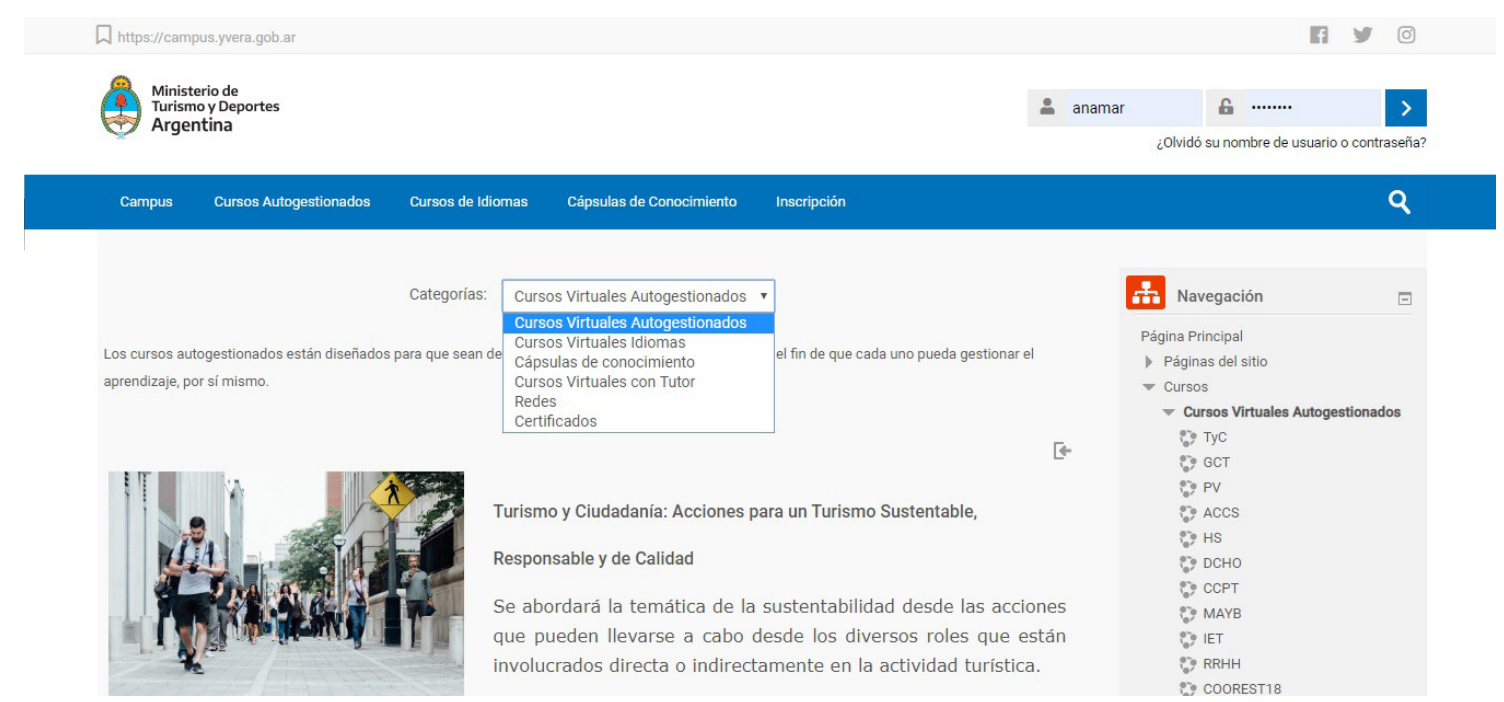

Figura 5. Cursos en Mintur. Fuente: https://campus.yvera.gob.ar/course/index.php?categoryid=4 
- Red Turismo Chile (https://redturismochile.cl/)

Fundada en 2011, se ha consolidado como consultora especializada en promoción turística y ha creado la red oficial de empresas y emprendimientos de turismo de Chile, en la cual las personas pueden conectar, de forma directa, con servicios de alojamiento, gastronomía, turismo, transporte, tiendas y cultura de todo el país. Además, destaca por sus servicios de desarrollo y gestión de proyectos, así como en la búsqueda y selección de personal para la industria turística nacional. El ser la principal plataforma de empleo en turismo del país la llevó a crear Turiwork (https:// turiwork.com/), la primera bolsa de empleo especializada en hotelería, turismo y gastronomía de Chile, espacio de reclutamiento laboral donde postulantes, empresas o instituciones, interactúan a partir de las ofertas y vacantes laborales.

Por el perfil de la red, la creación colectiva implica alta conectividad y alcanza gran asociación de alianzas y vínculos sociales exógenos; la producción de conocimiento es fundamental, ya que adquiere una reciprocidad sobresaliente en el intercambio de saberes, lo que fortalece la interacción dentro y fuera de ella.

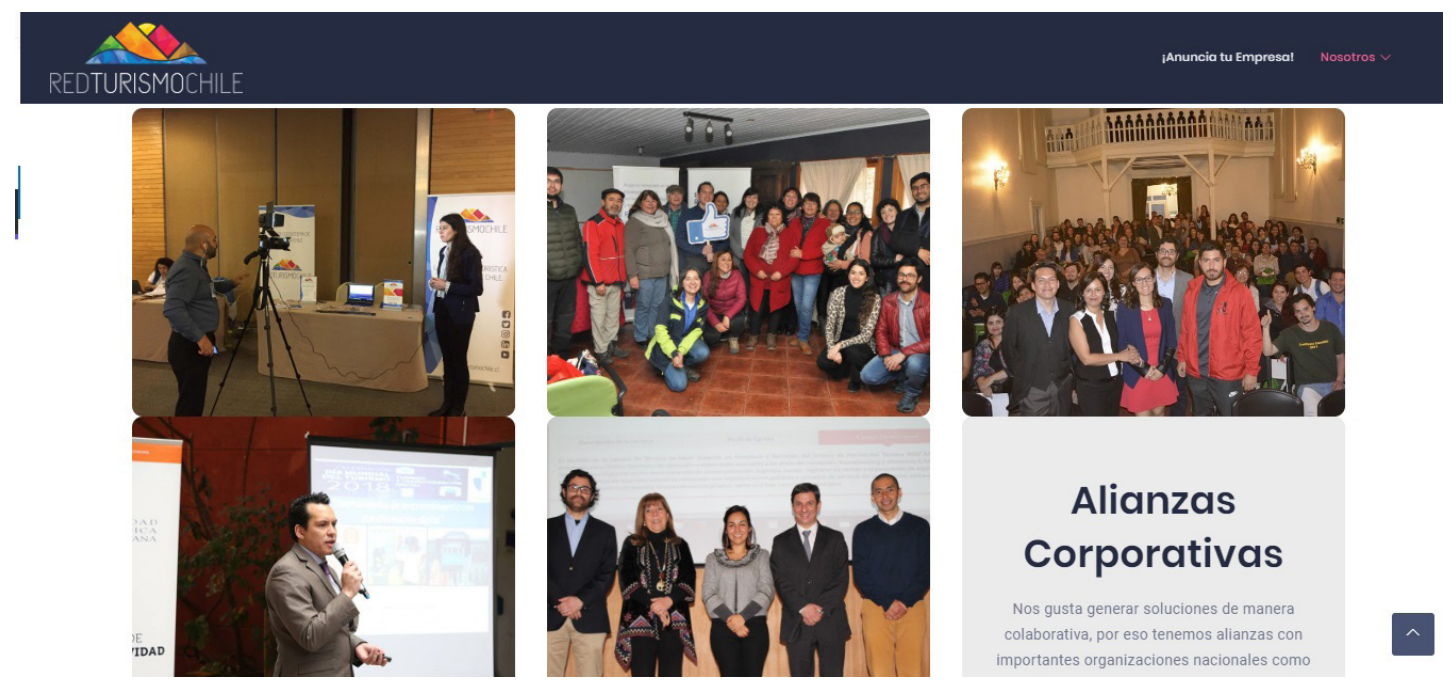

Figura 6. Alianzas de Red Turismo Chile. Fuente: https://redturismochile.cl/nosotros/ 
- Hosteltur (https://www.hosteltur.com)

Medio de "comunicación especializado en información turística profesional" (Hostetur, 2019, p. 2) que busca informar y formar a los profesionales del turismo al aportar información y conocimiento sobre las tendencias en este sector. Su penetración en este se ha incrementado gradualmente desde 2001 con su edición digital, lo que fortalece su interacción tanto en su plataforma como en redes sociales

El registro a la plataforma da acceso a webinars, ofertas de empleo, podcast, así como opiniones o notas de prensa creados por profesionales, empresarios, estudiantes o personas implicadas en el sector que comparten lo que crean y aprenden. Esto refleja la alta conectividad, participación en el desarrollo de conocimiento colaborativo y marcada reciprocidad en el intercambio de saberes, que origina una alta producción de conocimiento. Su asociación para el establecimiento de alianzas y la configuración exógena de vínculos sociales la han posicionado como un referente a nivel internacional al lograr una amplia participación y el reconocimiento por sus contenidos.

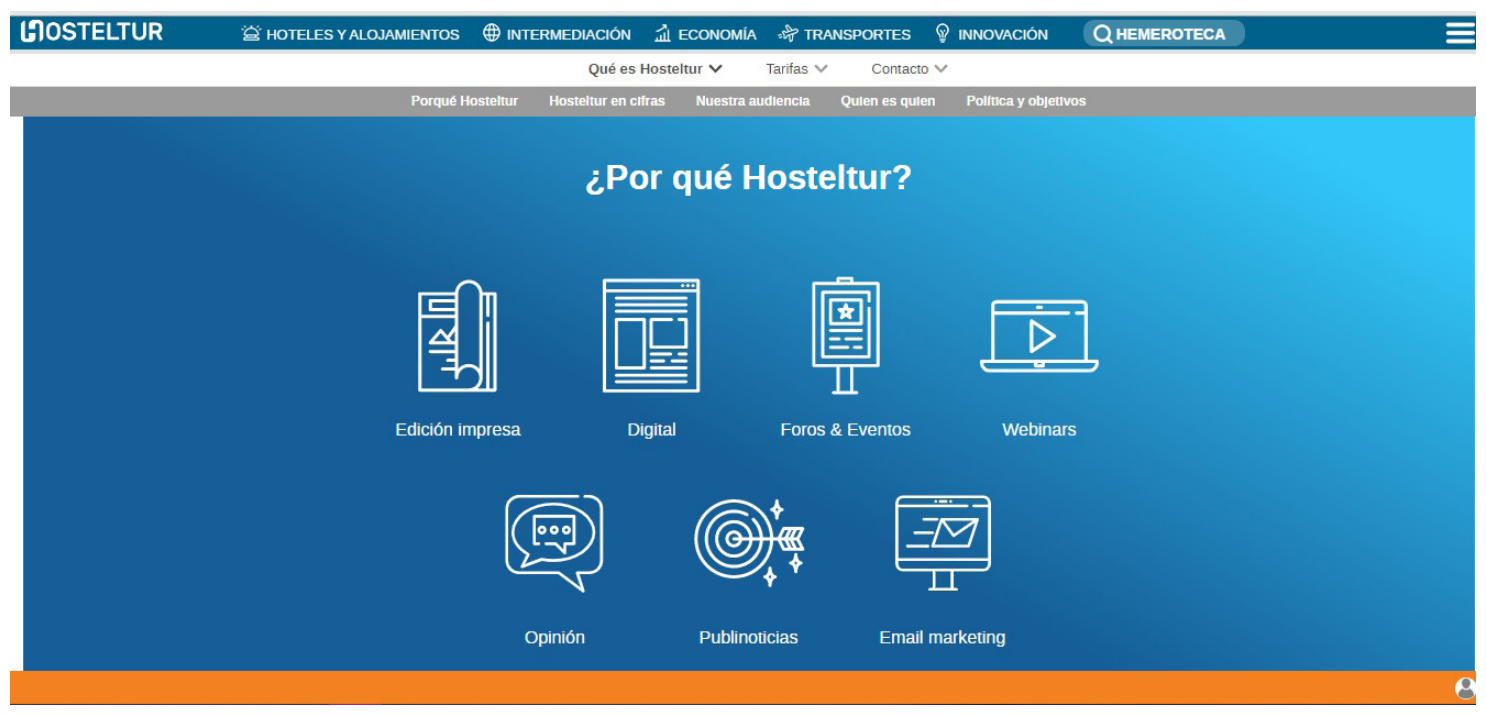

Figura 7. Contenido de Hosteltur. Fuente: https://www.hosteltur.com/dosier\#quien-es-quien 


\section{CONCLUSIONES: ESCENARIOS INTERCONECTADOS, TRANSFORMARSE PARA AFRONTAR RETOS}

Con el acercamiento a las comunidades observadas, reconocemos dos líneas de operación: por un lado, las redes que buscan la actualización y capacitación del individuo y, por otro, las que lo impulsan como productor y creador de conocimiento dentro de la colectividad.

Pudimos establecer también la relación entre las redes seleccionadas y reconocer los vínculos entre sus comunidades a través, principalmente, de la red social Twitter, la que se supone es la más accesible a sus seguidores. Derivado de la observación, trazamos un esquema de interacción (ver figura 8), el cual da cuenta de los enlaces creados entre las redes abordadas.

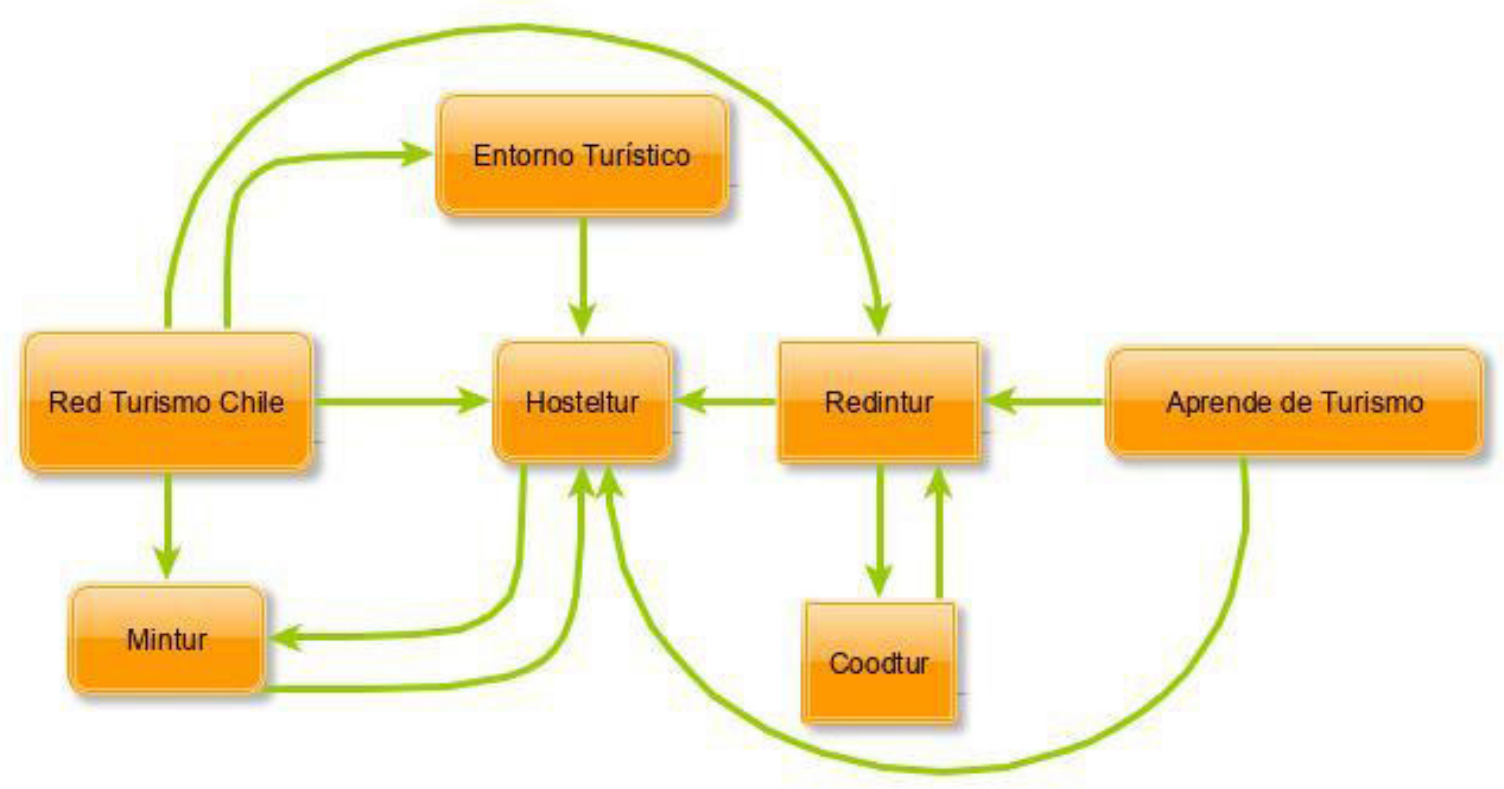

Figura 8. Esquema de interacción de redes en Twitter.

Hosteltur es un punto de referencia para el vínculo entre las redes y las comunidades seleccionadas; cinco de seis redes conocen su trabajo de manera directa y una en forma indirecta. Esta comunidad es un ejemplo de experiencia que ha transformado y favorecido los modos de aprender al generar conexiones y colaboración dentro de un entorno virtual sobre el interés común que es el turismo; comparte proyectos y conocimiento a partir de la cooperación e intercambio en beneficio mutuo de las personas implicadas en ella; así, resulta en una inteligencia colectiva; por ello, se posiciona como un referente para otras comunidades que se encuentran en crecimiento o las que necesitan estar actualizadas para ofrecer contenidos pertinentes de capacitación.

La conexión entre las redes permite el flujo de información vertida en sus plataformas, aspecto en el que juega un papel trascendental la Red Turismo Chile, pues es la cuenta que sigue a más redes. Redintur es la segunda más seguida, en contraste con Coodtur, lo cual no la limita a estar al tanto del trabajo de las otras comunidades, pues en Twitter las cuentas se reconocen y promueven su contenido al retuitear las publicaciones de otras redes. Este acercamiento a las comunidades observadas nos 
hace reflexionar que, mientras exista interacción, intercambio de saberes, configuración de vínculos sociales, producción y desarrollo de conocimiento colaborativo, estaremos frente a una red de aprendizaje que favorece formas de aprender.

Desde la posición de un individuo ávido de fortalecer su formación profesional en turismo en escenarios virtuales, todas estas redes le aportarán elementos para perfeccionar su formación y generarán un aprendizaje que ya es colaborativo, abierto, experimental, distribuido y por el bien común; esto trasciende hacia experiencias que habilitan su educación expandida al salir del entorno tradicional del aprendizaje.

Los desafíos para la formación en turismo se agudizan si no se busca una verdadera ruptura del paradigma tradicional del aprendizaje. Es insuficiente una educación híbrida o la llana implementación de cursos masivos en línea (Mooc) para considerar adentrarse a la dinámica de la cultura digital, y menos si estos cursos trabajan en la modalidad de xMooc y no como cMooc; de poco vale plantear un escenario distinto de aprendizaje si solo se traslada la educación tradicional a la virtualidad.

Sin embargo, esta idea no alcanza exclusivamente a organismos o instituciones encargados de una educación formal, sino al propio individuo involucrado en su proceso de formación, que debe ser consciente de las realidades a las que se enfrenta y ha de ser capaz de adaptarse a las circunstancias socioculturales y tecnológicas que están por presentarse; mientras no transite hacia otros espacios interconectados que impliquen colaboración, interacción e intercambio de saberes propios de la dinámica en la cultura digital, no logrará una transformación en su formación profesional y se convertirá en un agente pasivo.

Su labor no será fácil si consideramos los ilimitados volúmenes de información alojados en el ciberespacio; no todo el contenido albergado en la red suele aportar información de calidad, y la contaminación y saturación informativa obliga a discernir contenidos superfluos y basura que producen fatiga digital. En contraste, surge la idea de la zona red de aprendizaje, que alude a la red de coordinación social que tejemos con otros al aprender en internet (Suárez, 2018), de tal forma que el individuo puede plantearse cuestionamientos que lo orienten hacia el reconocimiento de su aprendizaje en un entorno virtual: por qué, para qué, cómo, dónde y con quién aprender en red. Sus propios intereses y componentes emocionales lo persuadirán para dar respuesta a estas interrogantes no sin olvidar las tendencias que marcan pauta en el sector.

En el turismo estas tendencias irrumpen fuertemente por la digitalización; algunas de ellas son la aparición de nuevas plataformas y aplicaciones que cambian la manera de negociar e interactuar en la actividad turística; los modelos disruptivos de intermediación que crean nuevos segmentos de mercado y habilitan relaciones antes no consideradas; la redefinición de las cadenas de valor y las demandas de los viajeros que apuntan hacia el valor experiencial y se perfilan como un turista creativo que genera su propio contenido y comparte en redes sociales.

Ante este escenario, observamos que las posibles transformaciones han de develarse para la formación profesional en turismo a partir de los cambios en la cultura digital y que, en consecuencia, inciden en el modo de aprender, en consideración de las siguientes circunstancias: 
-El mundo cada vez más interconectado obligará a los profesionales en turismo a desarrollar comunicación de doble vía por medio de redes de aprendizaje para potencializar lo que crea y aprende.

-No será suficiente la capacitación tradicional en línea (cursos estáticos); se tendrán que habilitar cursos que permitan la interacción y configuración de vínculos sociales con distintas personas para propiciar trabajo colaborativo.

-Las alianzas establecidas entre diversos sectores beneficiarán la educación expandida.

-La educación expandida tendrá nuevos escenarios. El desarrollo de tecnologías con realidad virtual y aumentada potencializará el aprendizaje a través de la experiencia dentro de escenarios virtuales.

- La digitalización de las cosas exigirá profesionales en turismo que tengan la capacidad de programar o automatizar sistemas relacionados con turismo.

-En atención a la experiencia personalizada del turista, serán indispensables desarrolladores de experiencias reales o virtuales.

De frente a los inminentes cambios de la formación profesional en turismo inmersa en la cultura digital, se articula la educación expandida y las redes de aprendizaje como formas de comunicación y gestión de conocimiento. Los enlaces simbólicos, interacciones, vínculos y transferencias representan procesos complejos que modifican la manera en que aprender lo complejo no radicará en la dificultad, sino en las conexiones que se manejan al interior de las redes.

Desde la perspectiva institucional, la transformación para la integración de las RA para el turismo comenzará (o continuará si es el caso), lo que revelará la pronta necesidad de configurar vínculos sociales exógenos e interacciones dinámicas que establezcan alianzas con diferentes sectores y deriven en el intercambio de saberes para una destacada producción de conocimiento con beneficio de las comunidades virtuales y presenciales ¿desde dónde? El primer paso será desde el aula, en un ejercicio decidido que conecte comunidades de aprendizaje con comunidades de práctica como lo plantea Caldeiro (2013) en su aportación en PENT Flacso, que reclama un estudio más específico para reconocer experiencias concretas y emprender nuevas dinámicas expansivas.

Tomar parte de una red a partir del acercamiento etnográfico virtual implicó identificar aquellas prácticas, y conexiones que dieran cuenta de cómo las personas están accediendo a ellas y acrecientan su proceso de formación profesional, ya que no es limitativa al ámbito escolar, porque las personas continúan capacitándose, colaborando y creando contenidos que benefician a un público interesado en turismo $\mathrm{y}$, al mismo tiempo, se favorecen de otros.

Las tendencias para el turismo puntean los nuevos roles de los profesionales inmersos en el área y comienzan a aparecer disrupciones que buscan dar solución a la llegada de retos y desafíos. Lo que queda es atender las nuevas demandas para la formación profesional en turismo que están surgiendo mientras se escriben estas líneas, todas ellas como resultado de las transformaciones digitales. 


\section{REFERENCIAS BIBLIOGRÁFICAS}

Álvarez, G. (2009). Etnografía virtual: exploración de una opción metodológica para la investigación en entornos virtuales de aprendizaje. Revista $Q$, vol. 3, núm. 6. Recuperado de https://revistas.upb.edu.co/index.php/revista_Q/article/view/7809

Amador, K., Arroyo, L. y Moo, M. D. J. (2018). Currículum y competencias genéricas de Tuning en estudiantes de turismo de la Universidad de Quintana Roo. Debates en Evaluación y Currículum/Congreso Internacional de Educación Currículum 2017, año 3, núm. 3, septiembre de 2017 a agosto de 2018. Recuperado de https://posgradoeducacionuatx.org/pdf2017/E200.pdf

Balakhadze, L. (2018). Los elementos del enfoque andragógico en la adquisición de la profesión turística en México. FILHA, vol. 9, núm. 11. Recuperado de http://revistas.uaz.edu.mx/index.php/filha/article/view/440

Barron, B. (2006). Interest and self-sustained learning as catalysts of development: A learning ecologies perspective. Human Development, núm. 49, pp. 193-224. doi: https://doi.org/10.1159/000094368

Burbules, C. (2012). El aprendizaje ubicuo y el futuro de la enseñanza. Encounters on Education, vol. 13. doi: https://doi.org/10.24908/eoe-ese-rse. v13i0.4472

Caldeiro, G. P. (2013). El aprendizaje en red y el trabajo colaborativo en entornos mediados por tecnología. Recuperado de http://www.pent.org.ar/institucional/publicaciones/aprendizaje-red-trabajo-colaborativo-entornos-mediados-por-tecnologia

Carrera, P. y Vallejo, E. (2015). Propuesta de un modelo educativo para la formación del talento humano en turismo en América Latina. Revista Latinoamericana de Turismología, vol. 1, núm. 2, pp. 30-41. Recuperado de https:// periodicos.ufjf.br/index.php/rlaturismologia/issue/view/378

Ceballos, C., Arias, C., Ruiz, A., Sanz, C. y Vázquez, I. (2010). La formación en turismo en España: pasado, presente y futuro en el Nuevo Espacio Europeo de Educación Superior. Cuadernos de Turismo, núm. 25, pp. 45-67. Recuperado de https://revistas.um.es/turismo/article/view/109571

Cobo, C. y Moravec, J. W. (2011). Aprendizaje invisible: hacia una nueva ecología de la educación. Transmedia XXI. Recuperado de http://www.razonypalabra. org.mx/varia/AprendizajeInvisible.pdf

Coll, C. (2013). El currículo escolar en el marco de la nueva ecología del aprendizaje. Aula de Innovación Educativa, núm. 219, pp. 31-36. Recuperado de https://www.researchgate.net/publication/255876239_El_curriculo_escolar_en_el_marco_de_la_nueva_ecologia_del_aprendizaje

Contreras, M. J., Juárez, J. y Ramírez, B. (2018). Evaluación de las competencias en el plan de estudios de tres licenciaturas en turismo en Puebla, México: percepciones de los estudiantes. RIDE. Revista Iberoamericana para la Investigación y el Desarrollo Educativo, vol. 9, núm. 17, pp. 665-687. doi: http:// dx.doi.org/10.23913/ride.v9i17.40

Dachary, A. C. y Arnaiz, F. C. (2016). Educación universitaria y turismo. Revista Latino-Americana de Turismología, vol. 2, núm. 2, pp. 8-17. doi: https://doi. org/10.34019/2448-198X.2016.v2.10019

Damm, L. (1998). Educación profesional en turismo. Gestión Turística, núm. 3, pp. 23-37. doi: https://doi.org/10.4206/gest.tur.1998.n3-02 
Deuze, M. (2006). Participation, remediation, bricolage: Considering principal components of a digital culture. The Information Society, núm. 22, pp. 63-75. doi: 10.1080/01972240600567170

Díaz, M. D. C y Quirós, F. J. (2013). El turismo en los nuevos planes de estudio universitarios. Una reflexión sobre la formación de los futuros profesionales del sector. Turismo e Innovación: VI Jornadas de Investigación en Turismo (pp. 127-147). Recuperado de https://idus.us.es/xmlui/bitstream/handle/11441/52960/diaz-fernandez.pdf?sequence=1\&isAllowed=y

Díaz, R. y Freire, J. (eds.) (2012). Educación expandida. Sevilla: ZEMOS98. Recuperado de http://www.zemos98.org/descargas/educacion_expandidaZEMOS98.pdf

Fernandes de Araújo, D. (2011). Formación profesional en turismo e inserción en el mercado laboral. Un estudio de caso del Polo Turístico Salvador/Bahía, Brasil, y su entorno. Estudios y Perspectivas en Turismo, vol. 20, núm. 1, pp. 57-74. Recuperado de http://www.estudiosenturismo.com.ar/PDF/V20/ v20n1a04.pdf

Fernández, A. (2015). Construyendo un sistema de aprendizaje a lo largo de la vida en turismo. España: Facultad de Ciencias Sociales y Humanas, Universidad de Deusto. Recuperado de http://cf.cdn.unwto.org/sites/all/files/pdf/ i.4.asunfernandezvillaran_0.pdf

Fonseca, A. (2011). Educación expandida y cultura digital. Una exploración de proyectos tecnosociales en Colombia. Revista Hallazgos, año 8, núm. 15, pp. 71-90. doi: https://doi.org/10.15332/s1794-3841.2011.0015.04

García, J. V. y Pérez, M. D. C. (2008). El grado en turismo: un análisis de las competencias profesionales. Cuadernos de Turismo, núm. 21, pp. 67-83. Recuperado de https://revistas.um.es/turismo/article/view/24991

Gómez, S. (2010). Repensar en el turismo: ante la irresistible tentación de lo pragmático y lo empírico. Anuario Turismo y Sociedad, vol. 9. Recuperado de https://revistas.uexternado.edu.co/index.php/tursoc/article/down$\operatorname{load} / 433 / 412 /$

Gómez, S. (2005) Los nuevos desafíos de la educación superior y de la investigación turística en México. Turismo y Sociedad, vol. 4, pp. 39-54. Recuperado de https://revistas.uexternado.edu.co/index.php/tursoc/article/view/2238

Groom, J. (2008). Murder, madness, mayhem is so EDUPUNK. Weblog bavatuesdays. Recuperado de http://bavatuesdays.com/murder-madness-mayhem/

Harasim, L., Hiltz, S. R., Turoff, M. y Teles, L. (2000). Redes de aprendizaje. Guía para la enseñanza y el aprendizaje en red. Barcelona: Gedisa.

Hine, C. (2004). Etnografía virtual (C. P. Hormazábal, trad.). Barcelona: Universidad Oberta de Cataluña.

Hosteltur (2019) Dossier de presentación. España. Recuperado de https://static.hosteltur.com/app/public/files/dosier-hosteltur-2019.pdf

Kereluik, K., Mishra, P., Fahnoe C. \& Terry, L. (2013). What knowledge is of most worth: Teacher knowledge for 21st Centyry Learning. Journal of Digital Learning in Teacher Education, vol. 29, núm. 4 Recuperado de https://files.eric. ed.gov/fulltext/EJ1010753.pdf

Lévy, P. (2007). Cibercultura. Informe al Consejo de Europa. Barcelona. Anthropos Editorial. 
Manassero, M. A., Ramis, C., Ramírez, E. y Guerra, L. E. (2016). Expectativas laborales y potencial directivo en estudiantes de licenciatura en Turismo. Retos de la Dirección, vol. 10, núm. 2, pp. 68-89. Recuperado de https://revistas. reduc.edu.cu/index.php/retos/article/view/151

Medina, L., Collado, M., Ortega, N. y Arango, A. (2012). TURed, un dispositivo de formación para el aprendizaje del turismo en redes de colaboración. Trabajo presentado en el XX Encuentro Internacional de Educación a Distancia. Guadalajara, México. Recuperado de http://www.udgvirtual.udg.mx/remeied/ index.php/memorias/article/view/131/47

Medina, S. y González, R. (2010). La formación en tecnologías de la información y de las comunicaciones (TIC) en la titulación de turismo: ¿es adecuada? Education in the Knowledge Society (EKS), vol. 11, núm. 1, pp. 371-388. Recuperado de http://revistas.usal.es/index.php/eks/article/view/6310

Montilla, A. D. J., Mora, L. y Guzmán, D. E. (2018). Modelo de aprendizaje y formación del profesional en turismo en la Universidad Laica Eloy Alfaro Demanabí. Revista Cognosis, vol. 3, núm. 2, pp. 35-44. doi: https://doi.org/10.33936/ cognosis.v3i2.1394

Osorio, M., Ramírez, I. y Viesca, C. (2017). Tendencias del turismo hasta 2030. Contrastes entre lo internacional y lo nacional. En Marta Nello Andreu y Alba Font Barnet (coords.). Anudar Red. Temas pendientes y nuevas oportunidades de cooperación en turismo (pp. 107-127). Recuperado de http://ri.uaemex. $\mathrm{mx} / \mathrm{handle} / 20.500 .11799 / 68788$

Organización Mundial de Turismo (2018a). El Consejo Ejecutivo de la OMT respalda las prioridades del secretario general para su mandato y a su nuevo equipo directivo. Noticia. Recuperado de https://media.unwto.org/es/pressrelease/2018-05-25/el-consejo-ejecutivo-de-la-omt-respalda-las-prioridades-del-secretario-gene

Organización Mundial del Turismo (2018b). La transformación digital y la innovación, protagonistas del Día Mundial del Turismo en 2018. Noticia. Recuperado de https://www2.unwto.org/es/press-release/2018-09-28/la-transformacion-digital-y-la-innovacion-protagonistas-del-dia-mundial-del

Rivas-Flores, J. I., Martínez, J. B., Valverde-Berrocoso, J. y Fernández-Rodríguez, J. B. (2018). Ecologías del aprendizaje en contextos múltiples. Análisis de proyectos de educación expandida y conformación de ciudadanía. Recuperado de https://riuma.uma.es/xmlui/handle/10630/16414

Sánchez, W. C. y Ortiz, P. A. (2017). La netnografía, un modelo etnográfico en la era digital. Revista Espacios, vol. 38, núm. 13. Recuperado de https://www. revistaespacios.com/a17v38n13/a17v38n13p28.pdf

Siemens, G. (2004) Conectivismo: una teoría de aprendizaje para la era digital. Traducción de Diego E. Leal Fonseca. Recuperado de http://clasicas.filos. unam.mx/files/2014/03/Conectivismo.pdf

Solórzano, F. y García, A. (2016). Fundamentos del aprendizaje en red desde el conectivismo y la teoría de la actividad. Revista Cubana de Educación Superior, vol. 35, núm. 3, pp. 98-112. Recuperado de http://www.rces.uh.cu/ index.php/RCES/article/view/139

Sterren, J. V. D. (2015). NHTV Breda University of Applied. Recuperado de Scienceshttp://cf.cdn.unwto.org/sites/all/files/pdf/jos_van_der_sterren_0.pdf

Suárez-Guerrero, C. (2018). Zona Red de Aprendizaje. En C. Cobo, S. Cortesi, L. Brossi, S. Doccetti, A. Lombana, N. Remolina, R. Winocur y A. Zucchetti (eds.). Jóvenes, transformación digital y formas de inclusión en América Latina (pp. 
123-132). Montevideo, Uruguay: Penguin Random House. Recuperado de https://digital.fundacionceibal.edu.uy/jspui/handle/123456789/229

Terrasa, D. (2018). ¿Qué es "Traveltech"? Recuperado de https://cursos.com/ que-es-traveltech/

Uribe, A. (2018). Concepto y prácticas de educación expandida: una revisión de la literatura académica. El Ágora USB, vol. 18, núm. 1, pp. 277-292. doi: https://doi.org/10.21500/16578031.3456

Uribe, A. (2017). Protoideas educativas de la educación expandida. Revista Virtual Universidad Católica del Norte, vol. 51, pp. 292-310. Recuperado de http://revistavirtual.ucn.edu.co/index.php/RevistaUCN/article/view/856

Uribe, A. (2015). Liberando el código: del monopolio cognitivo a la educación expandida. Revista de Investigaciones UCM, vol. 15, núm. 26, pp. 180-190. doi: http://dx.doi.org/10.22383/ri.v15i2.54

Vázquez, J. A. (2015). Nuevos escenarios y tendencias universitarias. Revista de Investigación Educativa, vol. 33, núm. 1, pp. 13-26. doi: http://dx.doi. org/10.6018/rie.33.1.211501 